\title{
FABRICATION AND CHARACTERIZATION OF PASSIVE MICROPUMP FOR MICROFLUIDICS BASED DEVICES
}

\author{
Prerna Balyan ${ }^{1,2}$, Deepika Saini ${ }^{1}$, Supriyo Das ${ }^{1}$, Payal Verma ${ }^{3}$, Ajay Agarwal ${ }^{1,2}$ \\ ${ }^{1}$ CSIR - Central Electronics Engineering Research Institute, Pilani, India \\ ${ }^{2}$ Academy of Scientific \& Innovative Research (AcSIR), New Delhi, India \\ ${ }^{3}$ Samara National Research University, Samara, Russia
}

\begin{abstract}
Point-of-care and low cost microfluidics platforms has found an accelerated research focus. One of the essential elements of microfluidics is driving liquid flow in microchannels. Device discussed gives a way to pump liquid passively through a microchannel. The present work represents fiber and paper based passive micro-pumping of liquids through microfluidic devices. The porous structure and network of capillaries inside the paper and fiber materials support spontaneous liquid movement. Agarose gel coating is used with paper in order to achieve variations flow rates. The effect of gel concentration on liquid flow is studied. The concept can be used ubiquitously for microfluidics device application for its low-cost and is feasible to integrate with devices for low resource settings.
\end{abstract}

Keywords: Microchannel, flow rate wicking.

Citation: Prerna Balyan, Deepika Saini, Supriyo Das, Payal Verma, Ajay Agarwal. Fabrication and characterization of passive micropump for microfluidics based devices. CEUR Workshop Proceedings, 2016; 1638: 159-165. DOI: 10.18287/1613-0073-2016-1638-159-165

\section{Introduction}

The research in the field of MEMS and Microfluidics is being explored extensively. With the successful development of devices such as microsensors [1-3], micro actuators, lab-ona-chip platforms, a transformation can be seen in the present technologies. Recently there has been extensive research on paper and fiber based microfluidics $[4,5]$. Paper and fiber material has been reported as a convenient substrate for plethora of microfluidics applications $[6,7]$. One of the essential elements of microfluidics is driving liquid flow in microchannels. Due to scaling effects a large pressure force is required to move fluids and/or suspended particles in microchannels. To move a fluid through microchannels 
various pumping mechanisms have been proposed in literature. A general classification divides the micro-pumping based on active and passive methods. Active methods such as electro-kinetic, electroosmotic, syringe pump, thermo-pneumatic etc. require external energy/power source. On the other hand, passive methods of micro-pumping of liquids utilize the surface properties and geometric effects at micro-scales such as capillary induced pumping or manual hand-held-syringe.

While inherent capillary action inside micro-channels makes one-time flow through the channel, or if the surface is wetting the capillary action will wet the channel spontaneously once but to make the liquid flow continuously some external force is required. Xiaoze et al. (2011) have proposed an evaporation-based Micropump and they have achieved constant and continuous flow rates of $\sim 100 \mathrm{nl} \mathrm{s}-1$. Researchers have used paper as the device substrate hence liquid flows automatically, however for other microfluidics platforms external or inbuilt micro pumping is required. Since materials with liquid absorption and wicking capabilities have shown huge potential to be used in microfluidics area, various modes to control and vary flow rates have been reported [8-11].

In present work the fluid flow is achieved via internal and external control. The internal control consists of coating of hydrophilic material layer onto the microchannel, whereas external control includes use of gel coated paper fixed in outlet to conduct a flow. The coated paper and fiber materials are used that caused a negative pressure generation to attain liquid flow in any microfluidic platform. Advantages offered by proposed method is its simple fabrication and low cost, wide availability of pumping material, uubiquitous use with broad range of flow rates achievable. Passive mode of operation eliminates the external power requirements as well as aapplicability to both open and closed channels.

\section{Material preparation and fabrication}

Desired flow rates are achieved by internal control and external control. In internal control, channel surface modification causes a self-driven flow of liquid. External control is achieved via connecting wicking material externally to the outlet of the microfluidic channel. Work presented here uses liquid absorbing materials like cotton fiber and cellulose paper to pump liquid through a polydimethylsiloxane (PDMS) elastomer based microchannel. The microchannel is fabricated in PDMS substrate via standard soft-lithography process, followed by plasma bonding of channel to glass substrate. For liquid pumping $1 \mathrm{~mm}$ x $20 \mathrm{~mm}$ wide Whatman filter paper strips were used as wicking substrate. The wicking strips were spin coated with Agarose gel.

The apparatus and method take advantage of the fact that liquids get absorbed/ wicked spontaneously in some special type of materials. The properties and makeup of material can be modified to obtain wide range of flow rates. A straight circular channel of $170 \mu \mathrm{m}$ width and $2 \mathrm{~cm}$ length made up of PDMS was used for the proof of concept. Other materials glass, $\mathrm{Si}$, Polymers, metals can also be used to fabricate the device. The outlet of the flowmeter was attached to the absorbing material and inlet was injected with the test solution. As the device does not require a fixture and tubes for flow injection, high magnification objectives could be used for the flow study. The 
liquid could flow continuously from inlet to outlet until the inlet was completely exhausted. Introduction of more flow controlling channels upstream and/or could help varying flow rates inside the main channel. The outlet wick creates a negative pressure differential causing a continuous liquid flow. It is necessary to prefill the channel. If channel is hydrophilic (glass or silicon dioxide channels) the micro-channel get wet spontaneously due to capillary action however a hydrophobic channel (PDMS) needs to be filled. A hand held syringe may be used for channel priming with liquid. Once the connection is established the sample analyte is introduced in the inlet reservoir. To measure flow rate either the liquid meniscus can be tracked or polymer beads may be inserted and tracked. A video is recorded and frames can be generated using any video analysis software. In the frames the beads/ meniscus crossing a graduation mark is noted down and liquid velocity and flow rate is measured. Various absorbing materials can be characterized for their liquid wicking ability by measuring the flow rate of the meniscus/beads.

The flowrate variations were obtained by varying shape, size and concentration of gel coating on the wicking strips. Different flow rates have been obtained at different concentrations (in wt. \%) of the agarose gel. The prepared liquid pumping material is inserted inside the output reservoir directly (Fig. 1). Small particles (micro-beads or blood cells) were added in very low concentrations and tracked for flow characterization. The flow was analyzed under optical microscope; the video was captured with a camera and was analyzed using ImageJ software. The method is very simple, cost effective for microfluidics platforms in point of care diagnostics for low resource settings applications.

\section{Results and discussion}

Paper and fiber based micropump carried out a continuous liquid flow through microchannels. Figure 1 depicts the overall scheme of liquid flow through a microchannel. The flow pumping material was inserted in the outlet section of the microchannel. The flow rate can be adjusted from very low to high value by changing the absorbing material type, material makeup/constituents, material geometry, material physical properties, material dimensions, and material assembly as well by devising new combinations of different materials.

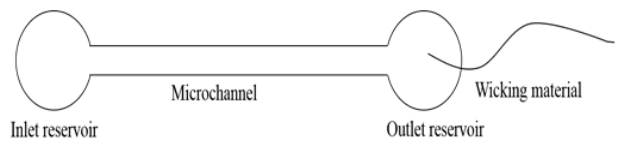

Fig. 1. Flow channel scheme showing Inlet and Outlet reservoir connected through microchannel and a wicking material placed in the outlet reservoir

In this work following methods are used to obtain flow rate variations:

Width of the wicking strips: By increasing the size/diameter of fiber significant flow rate variation was seen. Two sizes $1 \mathrm{~mm}$ x $20 \mathrm{~cm}$ and $5 \mathrm{~mm}$ x $20 \mathrm{~cm}$ wide wicking strips were used for conducting liquid flow. The velocity of particles was found to be 
greater in case of wider wicking strip, Fig. 2. The reason may be availability of higher surface area for wicking of liquid.

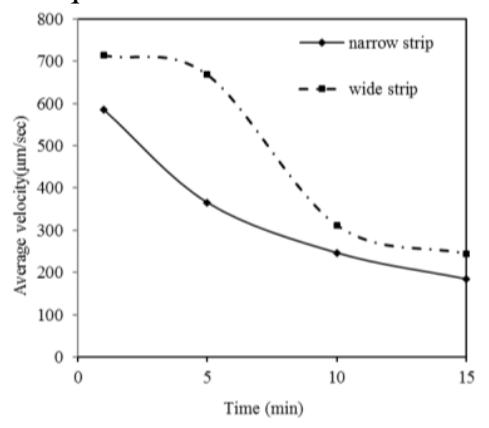

Fig. 2. Effect of width of wicking strip on solution flow. The flow rate is higher in wider sized strips than the narrow strips

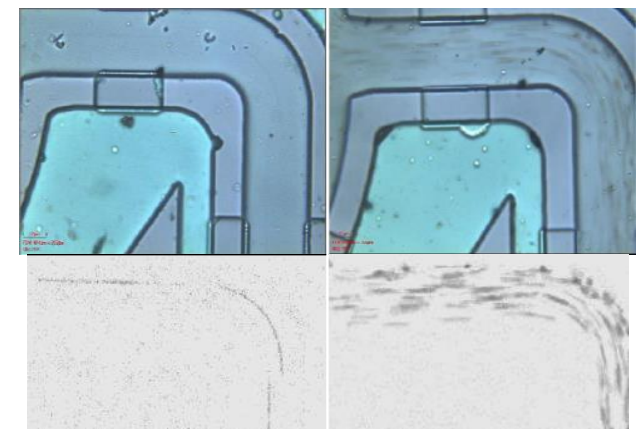

(a)

(b)

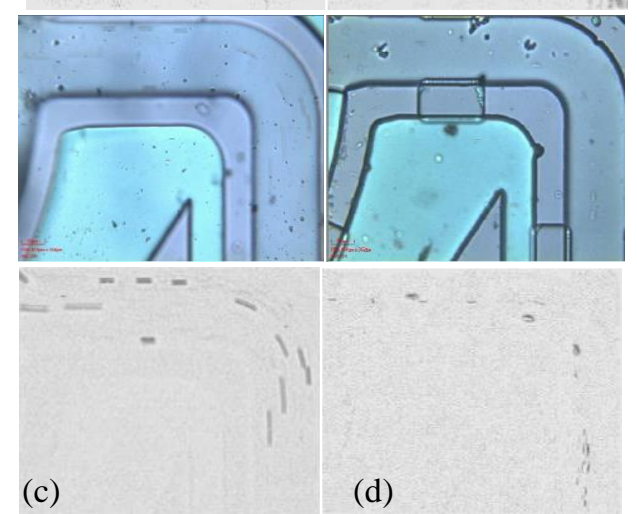

Fig. 3. Flow of polystyrene bead solution through the microchannel at different flow rates set up by coating the pumping material with different agarose gel concentrations. Particle trajectory is visualized via subtracting image background using ImageJ software, (a) Uncoated Wicking substrate, (b) Wicking substrate $+1 \%$ gel, (c) Wicking substrate $+5 \%$ gel and (d) Wicking substrate $+10 \%$. The flow rate decreases with the increase in gel concentration 
Agarose gel concentration: Agarose gel is a polymer with its constituent molecules connected with abundance of hydrogen bonds. The concentration of gel amounts to the pore size of the gel matrix which plays a crucial role in controlling the liquid flow. Very high flow rates were observed with untreated wicking material. Uniformly suspended polystyrene beads travelling at high speeds, appeared as long streaks during the liquid flow (Fig. 3(a)). With increasing the gel concentrations for the coating the beads travelled at slower rate (Fig. 3 (b)-(d)). This conforms to the potential of using Agarose gel to vary the liquid flow rate through microchannels. Figure 4 represents the average velocity of the tracked polystyrene beads through the microchannel.

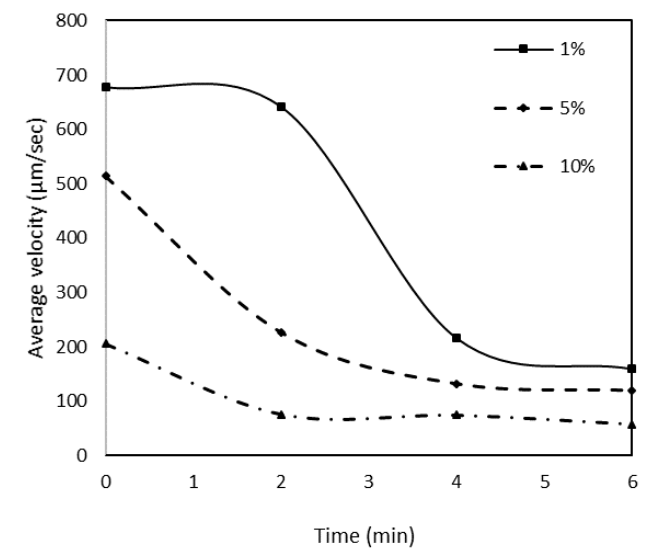

Fig. 4. Effect of gel concentration on solution flow
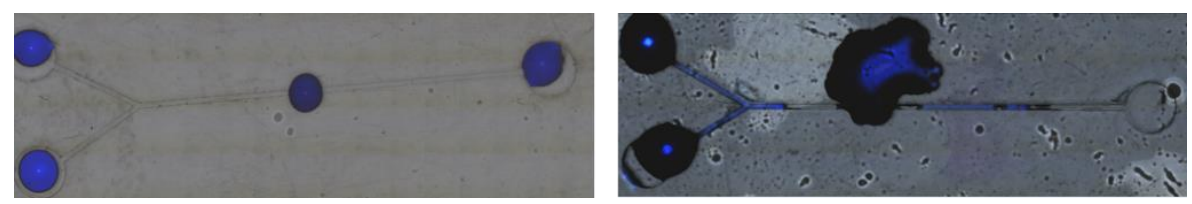

Fig. 5. Measured contact angle for (a) uncoated PDMS surface (Contact angle $106.4^{\circ}$ ) and (b) agarose coated PDMS surface (Contact angle $<40^{\circ}$ (solution spreads immediately)

Internal Control: PDMS is widely being used for microfluidics applications; however its hydrophobicity limits the spontaneous flow of liquid. Coating of agarose gel favors automatic flow of liquid through microchannel. The channel surface was spin coated with agarose gel (Fig. 5(b)). The non-coated surface had a contact angle around $106.5^{\circ}$ (Fig 5(a)), while the coated channel showed an immediate spread of liquid onto the surface (Fig. 5(b)). This coated layer of hydrogel causes immediate liquid wicking through the walls of the channel, and since channel acts as a fine capillary the wicking force generated at surface causes a bulk liquid flow.

In order to assess the conduction of flow. $1 \mu \mathrm{l}$ of colored solution was introduced into the inlets of coated and non-coated channels. A spontaneous liquid flow was observed through the coated channel (Fig. 6(b)). 
Figure 7 represents the snapshots of the liquid flow through channels at different time. The liquid filling in the inlet reservoir and outlet reservoir took longer time to fill due to bigger reservoir volumes. The liquid moved through the microchannel took $\sim 4 \mathrm{sec}$.
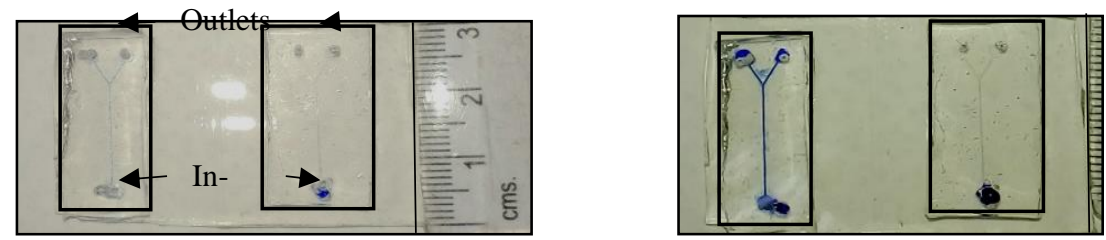

Fig. 6. (a) Fabricated coated and non-coated microchannels before sample introduction, (b) coated and non-coated channels after blue colour solution sample introduction. As a result of gel coating the sample flowed through the coated channel without any external field applied

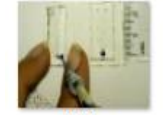

0.045

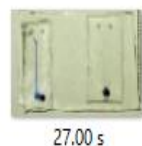

27.005

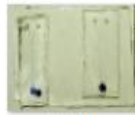

$20.00 \mathrm{~s}$

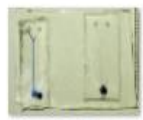

$28.00 \mathrm{~s}$

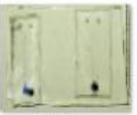

$22.88 \mathrm{~s}$

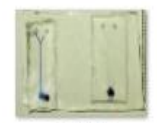

$29.00 \mathrm{~s}$

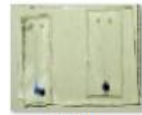

$23.00 \mathrm{~s}$

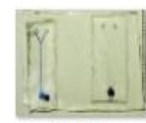

$30.00 \mathrm{~s}$

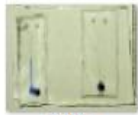

$24.00 \mathrm{~s}$

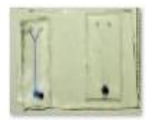

$31.00 \mathrm{~s}$

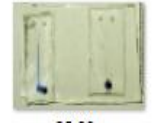

$25.00 \mathrm{~s}$

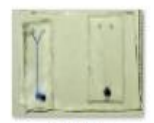

$32,00 \mathrm{~s}$

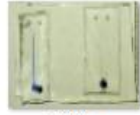

$26.00 \mathrm{~s}$

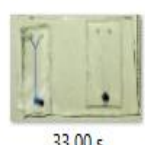

Fig. 7. Time sequenced snapshots of the flow of liquid through microchannel. The liquid flowed from inlet to outlet in the gel coated channel. No flow was observed in uncoated PDMS channel

\section{Conclusion}

The presented concept can be used to vary flow rates passively for microfluidics device application. Use of wicking material facilitates the spontaneous liquid flow through microchannels. The change in dimensions and surface energies of the wicking materials results in a wide variation in liquid flow characteristics. Flow rate increases with increased surface area of the material. Change in the surface coatings and pore area also affects the liquid flow. The liquid flow is observed to decrease with higher concentrations of the hydrophilic polymer coatings.

\section{References}

1. Verma P, Shekhar C, Arya SK, Gopal R. New design architecture of a 3-DOF vibratory gyroscope with robust drive operation mode and implementation. Microsystem Technologies, 2015; 21: 2175-2185.

2. Verma P, Gopal R, Arya SK. Analytical modeling and simulation of a 2-DOF drive and 1DOF sense gyro-accelerometer. Microsystem Technologies, 2013; 19(8): 1238-1249. 
3. Verma P, Arya SK, Gopal R. Lumped parameter analytic modeling and behavioral simulation of a 3-DOF MEMS gyro-accelerometer. Acta Mechanica Sinica, 2015; 31(6): 910919.

4. Martinez AW, Phillips ST, Whitesides GM, Carrilho E. Diagnostics for the developing world: microfluidic paper-based analytical devices. Analytical chemistry, 2009; 82(1): 310 .

5. Li X, Ballerini DR, Shen W. A perspective on paper-based microfluidics: current status and future trends. Biomicrofluidics, 2012; 6(1): 011301.

6. Yetisen AK, Akram MS, Lowe CR. Paper-based microfluidic point-of-care diagnostic devices. Lab on a Chip, 2013; 13(12): 2210-2251.

7. Lewis GG, DiTucci MJ, Phillips ST. Quantifying Analytes in Paper-Based Microfluidic Devices Without Using External Electronic Readers. Angewandte Chemie International Edition, 2012; 51(51): 12707-12710.

8. Shin JH, Park J, Park JK. Pressure-modulated flow control in paper-based microfluidic devices.

9. Jang I, Song S. Facile and precise flow control for a paper-based microfluidic device through varying paper permeability. Lab on a Chip, 2015; 15(16): 3405-3412.

10. Song H, Tice JD, Ismagilov RF. A microfluidic system for controlling reaction networks in time. Angewandte Chemie, 2003; 115(7): 792-796. 